\title{
EDITORIAL AND COMMENT \\ Advancing the Understanding of Social Determinants of Health Through Geospatial Analysis
}

\author{
Rebekah J. Walker, $P h D^{1,2,3}$, Brian Neelon, $P h D^{1,4}$, and Leonard E. Egede, MD, MS \\ ${ }^{1}$ Health Equity and Rural Outreach Innovation Center (HEROIC), Ralph H. Johnson Veterans Affairs Medical Center, Charleston, SC, USA; ${ }^{2} \mathrm{Center}$ for \\ Health Disparities Research, Medical University of South Carolina, Charleston, SC, USA; ${ }^{3}$ Department of Medicine, Division of General Internal \\ Medicine and Geriatrics, Medical University of South Carolina, Charleston, SC, USA; ${ }^{4}$ Department of Public Health Sciences, Medical University of \\ South Carolina, Charleston, SC, USA.
}

J Gen Intern Med 32(4):371-2

DOI: $10.1007 / \mathrm{s} 11606-016-3942-5$

(c) Society of General Internal Medicine 2016

$\mathrm{S}$ ocial determinants of health, such as neighborhood characteristics and residential segregation, are increasingly recognized as factors significantly influencing health outcomes and contributing to inequities in health. ${ }^{1,2}$ As health care systems are asked to address the social and environmental factors influencing their patients, a better understanding of the relationship between different social determinants of health and outcomes is necessary. In this issue of JGIM, Nelson et al. examine the association between neighborhood socioeconomic status (NSES) and all-cause mortality in a national sample of veterans. ${ }^{3}$ Using Veteran Health Administration (VHA) data on individual veteran characteristics and US Census Bureau data on census tract characterized NSES, Nelson et al. found those in the lowest (poorest) decile were 10\% more likely to die than those living in the highest (wealthiest) decile, after adjustment for socio-demographics, individual income, and comorbidities. $^{3}$ The authors should be commended for their analysis incorporating available spatial information and a national sample of veterans, addressing limitations of prior studies by using a population with racial/ethnic and income diversity and simultaneously adjusting for individual comorbidities and socioeconomic factors. ${ }^{3}$

Results from Nelson et al.'s study provide further evidence for inclusion of social determinants of health in decisions influencing provision of high-quality health care, such as risk adjustment and resource allocation. Additionally, it serves as an example of the increasing role spatial data analysis is playing in health services research and in understanding the role of social determinants. A wide variety of spatial analysis models and analytic frameworks are available to provide information about community needs and barriers to care and to provide guidance to policy makers as they consider how to allocate resources in the most cost-effective manner. ${ }^{4}$

Published online December 12, 2016
Although Nelson et al. obtain global estimates of the association between NSES and mortality while accounting for within-county correlation, the spatial random effect is used chiefly as a mechanism to account for this association. A natural extension of this work, expanding further into the field of spatial analysis, would be to examine spatial patterns in mortality across the study region after establishing a global effect of NSES. In fact, it might be of particular interest to explore whether the effect of NSES on mortality itself varies across space, through the inclusion of spatially varying regression coefficients for NSES, allowing investigators to identify areas where NSES has the most profound impact on mortality. ${ }^{5}$

We anticipate that spatial statistical methods will play an increasingly important role in the design and implementation of community-based efforts to improve access to care while reducing health costs. As seen in the paper by Nelson et al., the VA is well suited to conduct work using spatial analysis. As the largest managed care provider in the US, the VA has sufficient data to estimate subtle spatial effects with minimal bias. Moreover, given that the VA's research and policy efforts are closely aligned, findings can be quickly disseminated into practice. Additionally, the VA is in a position to directly inform community-based efforts to improve access to health care through resource allocation in VA hospitals and clinics, as well as through improved access to community providers via the Veterans Choice program. The ability to discern spatial patterns in health outcomes can facilitate design of effective interventions, inform outreach efforts, and ensure development of cost-efficient health services. ${ }^{4}$

There are a number of future directions for spatial and spatiotemporal analysis. These advanced spatial techniques, while computationally intensive, can provide significant advantages in translating results into action within health care systems. Spatiotemporal models can be used to investigate changing trends in the uptake of health services, such as ER visits. ${ }^{6,7}$ Likewise, spatial quantile regression and "two-part" models can provide insight into spatial patterns in health costs. ${ }^{8,9}$ These models can help distinguish subpopulations of individuals with unique patterns for utilization or expenditures for targeted interventions. Additionally, joint modeling of multiple health outcomes, for example, of utilization and 
illness, can provide insights into spatial connections not previously understood. ${ }^{4}$ Finally, spatial point process models can be used to study the regional distribution of health services and inform future allocation of resources. ${ }^{10}$ Understanding spatial and temporal trends will become increasingly important when planning community-based efforts, establishing new health centers, and targeting the needs of specific communities. ${ }^{4}$ Advanced spatial analytic techniques, such as those employed by Nelson et al. need to be more widely used and should be seen as one way to ensure the most comprehensive information is used by policy makers in their decisions.

Corresponding Author: Leonard E. Egede, MD, MS; Center for Health Disparities ResearchMedical University of South Carolina, 135 Rutledge Avenue, Room 280G, Charleston, SC 29425-0593, USA (e-mail:egedel@musc.edu).

\section{Compliance with ethical standards:}

Conflict of Interest: The authors have no financial disclosure or conflict of interest to report.

\section{REFERENCES}

1. Marmot M. Social determinants of health inequalities. Lancet. 2005;365:1099-104.

2. Williams DR, Collins C. Racial residential segregation: a fundamental cause of racial disparities in health. Public Health Rep. 2001;116:404-16.

3. Nelson K, Schwartz G, Hernandez S, Simonetti J, Curtis I, Fihn SD. The association of neighborhood environment and mortality: results from a national study of Veterans. JGIM. 2016. doi:10.1007/s11606-016-3905-x.

4. Neelon B. Spatial data analysis for health services research. In Lawson A, Banerjee S, Haining R, Ugarte L, eds. Handbook of Spatial Epidemiology, Chapter 29. Boca Raton: CRC Press; 2016: 549-562.

5. Gelfand AE, Kim H, Sirmans CF, Banerjee $\mathbf{S}$. Spatial modeling with spatially varying coefficient processes. J Am Stat Assoc. 2003;98(462):387-96.

6. Neelon B, Ghosh P, Loebs PF. A spatial Poisson hurdle model for exploring geographic variation in emergency department visits. J R Stat Soc Ser A. 2013;176:389-413.

7. Neelon B, Chang HC, Ling $\mathbf{Q}$, Hastings SN. Spatiotemporal hurdle models for zero-inflated count data: exploring trends in emergency department visits. Stat Methods Med Res. 2014; 1-19.

8. Neelon B, Li F, Burgette LF, Benjamin Neelon SE. A spatiotemporal quantile regression model for emergency department expenditures. Stat Med. 2015;34:2559-75.

9. Neelon B, Zhu L, Benjamin Neelon SE. Bayesian two-part spatial models for semicontinuous data with application to emergency department expenditures. Biostatistics. 2015;16:465-79.

10. Diggle JP. Statistical Analysis of Spatial Point Patterns. 3rd ed. Boca Raton: CRC Press; 2013. 